\title{
Negative pressure wound therapy: Potential publication bias caused by lack of access to unpublished study results data Frank Peinemann*1, Natalie McGauran ${ }^{1}$, Stefan Sauerland ${ }^{2}$ and Stefan Lange ${ }^{1}$
}

\author{
Address: ${ }^{1}$ Institute for Quality and Efficiency in Health Care (IQWiG), Dillenburger Str. 27, 51105 Cologne, Germany and ${ }^{2}$ Institute for Research \\ in Operative Medicine, University of Witten/Herdecke, Ostmerheimer Str. 200, 51109 Cologne, Germany \\ Email: Frank Peinemann* - frank.peinemann@iqwig.de; Natalie McGauran -n.mcgauran@iqwig.de; Stefan Sauerland - stefan.sauerland@ifom- \\ uni-wh.de; Stefan Lange - stefan.lange@iqwig.de \\ * Corresponding author
}

Published: II February 2008

BMC Medical Research Methodology 2008, 8:4 doi:10.1 I86/147/-2288-8-4
Received: 2 May 2007

Accepted: II February 2008

This article is available from: http://www.biomedcentral.com/I47I-2288/8/4

(c) 2008 Peinemann et al; licensee BioMed Central Ltd.

This is an Open Access article distributed under the terms of the Creative Commons Attribution License (http://creativecommons.org/licenses/by/2.0), which permits unrestricted use, distribution, and reproduction in any medium, provided the original work is properly cited.

\begin{abstract}
Background: Negative pressure wound therapy (NPWT) is widely applied, although the evidence base is weak. Previous reviews on medical interventions have shown that conclusions based on published data alone may no longer hold after consideration of unpublished data. The main objective of this study was to identify unpublished randomised controlled trials (RCTs) on NPWT within the framework of a systematic review.

Methods: RCTs comparing NPWT with conventional wound therapy were identified using MEDLINE, EMBASE, CINAHL and The Cochrane Library. Every database was searched from inception to May 2005. The search was updated in December 2006. Reference lists of original articles and systematic reviews, as well as congress proceedings and online trial registers, were screened for clues to unpublished RCTs. Manufacturers of NPWT devices and authors of conference abstracts were contacted and asked to provide study information. Trials were considered nonrandomised if concealment of allocation to treatment groups was classified as "inadequate". The study status was classified as "completed", "discontinued", "ongoing" or "unclear". The publication status of completed or discontinued RCTs was classified as "published" if a full-text paper on final study results (completed trials) or interim results (discontinued trials) was available, and "unpublished" if this was not the case. The type of sponsorship was also noted for all trials.
\end{abstract}

Results: A total of 28 RCTs referring to at least 2755 planned or analysed patients met the inclusion criteria: 13 RCTs had been completed, 6 had been discontinued, 6 were ongoing, and the status of 3 RCTs was unclear. Fulltext papers were available on $30 \%$ of patients in the 19 completed or discontinued RCTs (495 analysed patients in 10 published RCTs vs. II54 planned patients in 9 unpublished RCTs). Most information about conference abstracts and unpublished study information referring to trials that were unpublished at the time these documents were generated was obtained from the manufacturer Kinetic Concepts Inc. (KCl) (I9 RCTs), followed by The Cochrane Library (I8) and a systematic review (I5). We were able to obtain some information on the methods of unpublished RCTs, but results data were either not available or requests for results data were not answered; the results of unpublished RCTs could therefore not be considered in the review. One manufacturer, $\mathrm{KCl}$, sponsored the majority of RCTs $(19 / 28 ; 68 \%)$. The sponsorship of the remaining trials was unclear.

Conclusion: Multi-source comprehensive searches identify unpublished RCTs. However, lack of access to unpublished study results data raises doubts about the completeness of the evidence base on NPWT. 


\section{Background}

Conservative estimates report that about $1 \%$ of the population in Western countries is affected by chronic wounds [1], with a much higher prevalence rate in inpatient and nursing facilities [2]. Besides presenting a significant risk factor for complications such as infection and amputation, chronic wounds also lead to marked impairment of patients' quality of life $[3,4]$.

Negative pressure wound therapy (NPWT) consists of an open-cell foam dressing covered with an adhesive drape. The dressing is connected to a vacuum pump that creates and maintains a subatmospheric pressure [5]. The most commonly used NPWT device is the Vacuum Assisted Closure (V.A.C. ${ }^{\circledast}$ ) device.

The Federal Joint Committee (Gemeinsame Bundesausschuss), the decision-making body of the self-administration of the German health care service, commissioned the Institute for Quality and Efficiency in Health Care (Institut für Qualität und Wirtschaftlichkeit im Gesundheitswesen, $I Q W i G)$ to conduct a systematic review on the efficacy and safety of NPWT versus conventional wound therapy in patients with acute or chronic wounds. The full review was published in German in March 2006, and an English-language article has recently been published [6,7]. An update of the systematic review, a rapid report, was published in German in January 2007 [8].

Although NPWT is widely applied, particularly for chronic wounds, at the time of the review only a few relevant randomised controlled trials (RCTs) were available; these were, moreover, of poor quality [9]. Previous reviews on NPWT have not provided clear evidence of the superiority of NPWT over conventional wound therapy. For example, a review published by the Canadian Medical Advisory Secretariat in 2006 concluded that "Based on the evidence to date, the clinical effectiveness of NPWT to heal wounds is unclear" [10].

Previous reviews on medical interventions have shown that conclusions on efficacy and safety based on published data alone may no longer hold after consideration of unpublished data, which may reverse favourable riskbenefit profiles and attenuate treatment effects of an apparently superior intervention [11-13]. Within the framework of the systematic review on NPWT, our main objective was therefore not only to identify published, but also unpublished completed or discontinued RCTs in order to gain as complete an overview as possible of the evidence on NPWT. Further aims were to locate ongoing RCTs for potential consideration in future updates, and to determine the type of sponsorship of all eligible trials.

\section{Methods}

The literature search comprised a total of 7 steps. Table 1 shows an overview of the sources used.

We searched 4 bibliographic databases (MEDLINE, EMBASE, CINAHL, and The Cochrane Library) for RCTs on NPWT versus conventional wound therapy in patients with acute or chronic wounds. All databases were searched from inception to May 2005. The search was updated in December 2006 within the framework of the preparation of the rapid report on NPWT [8]. The search strategy in MEDLINE (Table 2) was applied according to the Cochrane Highly Sensitive Search Strategy described in the Cochrane Handbook for Systematic Reviews of Interventions [14], and tailored to the requirements of each database. Other search strategies are available upon request.

We then screened online trial registers (Table 1) and the US Food and Drug Administration website, and contacted German authorities, requesting information on all available RCTs on NPWT. Prior to the start of the review, the Federal Joint Committee had asked experts to provide relevant literature; these citations were also obtained by IQWiG as part of the commission. Moreover, the reference lists of retrieved original articles and systematic reviews, as well as congress proceedings, were handsearched.

Two manufacturers of NPWT devices were identified: Kinetic Concepts Inc. (KCI, San Antonio, Texas, USA) markets the V.A.C. ${ }^{\circledR}$ device, and BlueSky Medical Group Inc. (BSM, Carlsbad, California, USA) markets the Versatile 1 Wound Vacuum System ${ }^{\oplus}$. Both were asked to provide information on the study status and publication status of sponsored trials, as well as on methods and results. Finally, we contacted the authors of conference abstracts by e-mail and by post. All contact details of the relevant institutions were checked beforehand on the corresponding websites. Manufacturers and authors were informed that IQWiG does not accept "commercial in confidence" data and publishes all data contributing to a systematic review [15].

All languages were included, as long as a title was available in English. If the title indicated a potential relevance of the study, the corresponding article was obtained and translated. Only RCTs comparing NPWT versus conventional therapy were eligible. Trials were considered nonrandomised if concealment of allocation to treatment groups was classified as "inadequate" [16]. The intervention was classified as NPWT if a medical device system identical or comparable to the V.A.C. ${ }^{\circledR}$ Therapy System was used. 
Table I: Literature sources and search steps*

\begin{tabular}{ll}
\hline Category & Sources \\
\hline $\begin{array}{l}\text { Step I } \\
\text { Bibliographic databasest }\end{array}$ & \\
& MEDLINE: Includes PubMed and Clinical Queries. \\
& EMBASE \\
& The Cochrane Library. These databases were searched: \\
& Cochrane Database of Systematic Reviews (CDSR; Cochrane Reviews) \\
& Database of Abstracts of Reviews of Effects (DARE; Other Reviews) \\
& Cochrane Central Register of Controlled Trials (CENTRAL; Clinical Trials) \\
& Health Technology Assessment Database (HTA; Technology Assessments) \\
& NHS Economic Evaluation Database (NHSEE; Economic Evaluations) \\
& CINAHL
\end{tabular}

\section{Step 2}

Online trial registers

ClinicalTrials.gov

National Research Register (NRR)

Step 3

Authorities
German Federal Joint Committee (references cited by experts in 2003)

United States Federal Drug Administration (FDA) (online search)

German Federal Institute for Drugs and Medical Devices (direct enquiry by e-mail and phone)

\section{Step 4}

Systematic reviews $\ddagger$
Ontario Health Technology Advisory Committee 2006 (OHTAC)

Pham 2006, Australian Safety and Efficacy Register of New Interventional Procedures Surgical (ASERNIP-S)

Costa 2005, McGill University Health Centre Technology Assessment Unit (MUHC)

Samson 2004, United States Agency for Healthcare Research and Quality (AHRQ)

Higgins 2003, Centre for Clinical Effectiveness (CCE)

Fisher 2003, Canadian Coordinating Office of Health Technology Assessment (CCOHTA)

Evans 200I, Cochrane database of systematic reviews (CDSR)

\section{Step 5}

Congress proceedings

V.A.C. ${ }^{\circledR}$ Wundtherapie, 10 Jahre V.A.C., Drei-Länder-Kongress [V.A.C. Wound Therapy, 10 Years of V.A.C., Three-Country Conference], Graz, Austria; 2005. Symposium on Advanced Wound Care, San Diego, California, USA; 2005.

Second World Union of Wound Healing Societies Meeting, Paris, France; 2004.

V.A.C. ${ }^{\circledR}$ Wundtherapie, Drei-Länder-Kongress [V.A.C. Wound Therapy, Three-Country Conference], Mainz, Germany; 2004.

First international topical negative pressure (TNP) therapy focus group meeting in London, UK, December 2003. Proceedings. Edited by: Banwell P, Teot L. Supported by the European Tissue Repair Society. Faringdon, UK: TXP Communications; 2004.

Vacuum Assisted Closure (V.A.C. $\left.{ }^{\circledR}\right)$, Salzburg, Austria; 2003.

Eleventh Annual Meeting and Educational Symposium, Wound Healing Society, Albuquerque, New Mexico, USA; 2001.

\section{Step 6}

Manufacturers (direct enquiries by e-mail and post) Kinetic Concepts, Inc., San Antonio, Texas, USA.

BlueSky Medical Group, Inc., Carlsbad, California, USA

\section{Step 7}

Authors (direct enquiries by e-mail and post)

Adams; Armstrong; Bayer; Foo; Fryer; Greer; Gupta; Heath; Lantis; McCarthy J; McCarthy M; Molnar; Niezgoda; Orgill; Payne; Stannard (3 RCTs); Vuerstaek; Walker

\footnotetext{
* Other sources available on request.

† Search executed in May 2005 and updated in December 2006.

$\ddagger$ Hayes 2003 [7I] was not publicly available and therefore not considered. OHTAC 2004 [72] and Pham 2003 [73] were not considered because updated versions are available.
}

Results of trials that were not available as full-text publications were only considered in the review if detailed data 
Table 2: Search strategy applied in MEDLINE

\begin{tabular}{|c|c|}
\hline No. & Search terms \\
\hline I & amputation\$.ti,ab. \\
\hline 2 & exp AMPUTATION/ \\
\hline 3 & exp AMPUTATION TRAUMATICI \\
\hline 4 & burn $\$ . t i, a b$. \\
\hline 5 & $\exp$ BURNS/ \\
\hline 6 & decubit\$.ti,ab. \\
\hline 7 & deglov\$.ti,ab. \\
\hline 8 & diabet\$.ti,ab. \\
\hline 9 & exp DIABETES MELLITUS/ \\
\hline 10 & electric $\$$ injur $\$ . t i, a b$. \\
\hline 11 & frostbit\$.tw. \\
\hline 12 & exp FROSTBITE/ \\
\hline 13 & laceration\$.ti,ab. \\
\hline 14 & exp LACERATIONS/ \\
\hline 15 & open-abdom $\$ . t i, a b$. \\
\hline 16 & $\exp A B D O M I N A L$ WALL/su \\
\hline 17 & plastic-surg\$.ti,ab. \\
\hline 18 & exp SURGERY, PLASTIC/ \\
\hline 19 & reconstruct $\$$-surg $\$$. ti,ab. \\
\hline 20 & exp RECONSTRUCTIVE SURGICAL PROCEDURES/ \\
\hline 21 & skin-graft\$.ti,ab. \\
\hline 22 & skin-transplant $\$ . t i, a b$. \\
\hline 23 & $\exp$ SKIN TRANSPLANTATION/ \\
\hline 24 & surg\$ flap.ti,ab. \\
\hline 25 & exp SURGICAL FLAPS/ \\
\hline 26 & thermal injur $\$ . t i, a b$. \\
\hline 27 & exp ELECTRIC INJURIES/ \\
\hline 28 & ulcer\$.ti,ab. \\
\hline 29 & ul\#us\$.ti,ab. \\
\hline 30 & $\exp$ SKIN ULCER/ \\
\hline 31 & exp SOFT TISSUE INFECTIONS/ \\
\hline 32 & $\exp$ ULCER/ \\
\hline 33 & wound\$.ti,ab. \\
\hline 34 & $\exp$ WOUND INFECTION/ \\
\hline 35 & exp WOUND HEALING/ \\
\hline 36 & wound dehiscence.ti,ab. \\
\hline 37 & exp SURGICAL WOUND DEHISCENCE/ \\
\hline 38 & "mini-v.a.c.\$".ti,ab. \\
\hline 39 & negative-pressur $\$ . t i, a b$. \\
\hline 40 & subatmospheric-pressur $\$ . t i, a b$. \\
\hline 41 & sub-atmospheric-pressur $\$ . t i, a b$. \\
\hline 42 & \$suction\$.ti,ab. \\
\hline 43 & $\exp$ SUCTION/ \\
\hline 44 & vacuum $\$ . t i, a b$. \\
\hline 45 & $\exp$ VACUUM/ \\
\hline 46 & (clin $\$$ adj25 trial\$).ti,ab. \\
\hline 47 & clinical trial.pt. \\
\hline 48 & $\exp$ CLINICAL TRIALS/ \\
\hline 49 & (control\$ or prospectiv\$ or volunteer\$).ti,ab. \\
\hline 50 & controlled clinical trial.pt. \\
\hline 51 & COMPARATIVE STUDY.sh. \\
\hline 52 & DOUBLE BLIND METHOD.sh. \\
\hline 53 & exp EVALUATION STUDIES/ \\
\hline 54 & FOLLOW-UP STUDIES.sh. \\
\hline 55 & placebo\$.ti,ab. \\
\hline 56 & PLACEBOS.sh. \\
\hline 57 & PROSPECTIVE STUDIES.sh. \\
\hline 58 & random $\$ . t i, a b$. \\
\hline 59 & randomized controlled trial.pt. \\
\hline
\end{tabular}


Table 2: Search strategy applied in MEDLINE (Continued)

\begin{tabular}{ll}
\hline 60 & RANDOM ALLOCATION.sh. \\
61 & RANDOMIZED CONTROLLED TRIALS.sh. \\
62 & RESEARCH DESIGN.sh. \\
63 & SINGLE BLIND METHOD.sh. \\
64 & $(($ singl\$ or doubI\$ or trebl\$ or tripI\$) adj25 (blind $\$$ or mask $\$)$ ).ti,ab. \\
65 & or/l-37 \\
66 & or $/ 38-45$ \\
67 & or $/ 46-65$ \\
68 & and $/ 66-68$ \\
\hline
\end{tabular}

Note: We have amended or deleted 2 terms contained in the original strategy. The first term "frostbite $\$ . t i, a b . "$ was replaced by "frostbit $\$ . t w . "$. The second term "(metaanaly\$ or (meta and analy\$) or ((review or search\$) and (systemat $\$))$ ).ti,ab." was deleted. The amended strategy did not affect the search results. However, as an article may be published in the future and missed by not truncating the first term at the appropriate spot, the term "frostbit\$.tw." should be used. The second term is superfluous.

were available (e.g. from a clinical study report or a manuscript in press). Results data from conference abstracts were not considered.

All steps of the literature screening were performed by two reviewers independently of one another. Any disagreements were resolved by discussion. The titles and abstracts of the retrieved documents were screened to exclude citations that were clearly irrelevant. The full texts of the remaining potentially relevant articles were then screened to identify RCTs that fulfilled the inclusion criteria stated above.

With the help of the information obtained, the study status of the RCTs identified was classified as "completed", "discontinued", "ongoing" or "unclear". The terminology used in the literature to classify unpublished data or socalled "grey literature" is inconsistent [12]. We classified the publication status of completed or discontinued trials as "published" if a full-text paper on final study results (completed trials) or interim results (discontinued trials) was identified in the literature search, and "unpublished" if this was not the case. In addition, for ongoing trials it was indicated whether interim results were available or not. The type of sponsorship was also noted for all trials.

Only a summary of results on the quality assessment and outcomes of the RCTs included in the IQWiG systematic review and rapid report is presented here, as the main focus of this paper was to identify unpublished RCTs.

\section{Results}

An overview of the search results is presented in Table 3. Detailed information on identified published and unpublished RCTs, as well as on the RCTs included in the systematic review and rapid report, is presented in Table 4.

\section{Search results}

Of 2675 potentially relevant publications, 317 full-text papers were obtained for further assessment. Of these, 289 were excluded as not relevant (Figure 1).

A total of 28 RCTs on NPWT referring to at least 2755 planned or analysed patients were identified: 13 RCTs (801 patients; 29\%) had been completed [17-29], 6 RCTs (848 patients; 31\%) had been discontinued [30-35], 6

Table 3: Overview of study status, publication status, and sponsorship (Status: December 2006)

\begin{tabular}{|c|c|c|c|c|c|}
\hline & \multicolumn{4}{|c|}{ Study status } & \multirow[b]{2}{*}{ Total (trials/patients) } \\
\hline & $\begin{array}{l}\text { Completed (trials/ } \\
\text { patients) }\end{array}$ & $\begin{array}{c}\text { Discontinued (trials/ } \\
\text { patients) }\end{array}$ & $\begin{array}{l}\text { Ongoing (trials/ } \\
\text { patients) }\end{array}$ & $\begin{array}{l}\text { Unclear (trials/ } \\
\text { patients) }\end{array}$ & \\
\hline \multicolumn{6}{|c|}{ Publication status } \\
\hline Published* & $9 / 473$ & $1 / 22$ & $(2 / 88)$ & $(0 / 0)$ & $10 / 495 \dagger$ \\
\hline Unpublished $\ddagger$ & $4 / 328$ & $5 / 826$ & $(4 / 500)$ & $(3 / ?)$ & $9 / 1154 \dagger$ \\
\hline \multicolumn{6}{|l|}{ Sponsorship } \\
\hline Industrial & $8 / 413$ & $6 / 848$ & $5 / 1076$ & $0 / 0$ & $19 / 2337$ \\
\hline Unclear & $5 / 388$ & $0 / 0$ & $1 / 30$ & $3 / ?$ & $9 / 418$ \\
\hline Total & $|3 / 80|$ & $6 / 848$ & $6 / 1106$ & $3 / ?$ & $28 / 2755$ \\
\hline
\end{tabular}

* Analysed patients.

† Published/unpublished: full-text publications of final results (completed trials) or interim results (discontinued trials) were available/not available. $\ddagger$ Planned patients. 
Table 4: Search results for randomised controlled trials on negative pressure wound therapy* (Status: December 2006)

\begin{tabular}{|c|c|c|c|c|c|c|c|c|c|}
\hline Study status & No. & Abstract & $\begin{array}{l}\text { Information source } \\
\text { for abstract }\end{array}$ & Sponsor (ID $t$ ) & $\begin{array}{l}\text { Reply to inquiry to } \\
\text { sponsor or author }\end{array}$ & $\begin{array}{l}\text { Patients } \\
\text { planned } ¥\end{array}$ & $\begin{array}{l}\text { Patients ana- } \\
\text { lysed } \S\end{array}$ & $\begin{array}{l}\text { Results data } \\
\text { available }\end{array}$ & $\begin{array}{l}\text { Full-text publica- } \\
\text { tion availablell }\end{array}$ \\
\hline \multicolumn{10}{|l|}{ Completed } \\
\hline & I & $\begin{array}{c}\text { Vuerstaek } \\
\text { [74] }\end{array}$ & WUWHS 2004 & $\mathrm{KCl}(\mathrm{VAC} \mathrm{VLU})$ & $\mathrm{KCl}$ & - & 60 & Yest & Vuerstaek 2006 [23] \\
\hline & 2 & No abstract & - & Unclear & - & - & 60 & Yes $\pi$ & Llanos 2006 [25] \\
\hline & 3 & Obdeijn [75] & WUWHS 2004 & $\mathrm{KCl}(\mathrm{No} \mathrm{ID})$ & $\mathrm{KCl}$ & - & 65 & Yest & $\begin{array}{c}\text { Braakenburg } 2006 \\
{[2 \mathrm{I}]}\end{array}$ \\
\hline & 4 & Payne [76] & WUWHS 2004 & $\begin{array}{c}\mathrm{KCl} \text { (VAC } 200 \mathrm{I}- \\
07)\end{array}$ & $\mathrm{KCl}$; author & - & 162 & Yes\# & Armstrong 2005 [22] \\
\hline & 5 & Moues [77] & WUWHS 2004 & $\begin{array}{l}\mathrm{KCl}(\mathrm{PMID} \\
\mathrm{I} 4974959)\end{array}$ & $\mathrm{KCl}$ & - & 54 & Yes\# & Moues 2004 [20] \\
\hline & 6 & Heath [78] & The Cochrane Library & $\begin{array}{l}\mathrm{KCl}(\mathrm{PMID} \\
\mathrm{I} 5468399)\end{array}$ & $\mathrm{KCl}$; author & - & 20 & Yes\# & Moisidis 2004 [18] \\
\hline & 7 & No abstract & - & $\begin{array}{l}\mathrm{KCl}(\mathrm{PMID} \\
\mathrm{I} 4534844)\end{array}$ & $\mathrm{KCl}$ & - & 6 & Yes\# & Eginton 2003 [17] \\
\hline & 8 & No abstract & - & $\begin{array}{l}\mathrm{KCl}(\mathrm{PMID} \\
\mathrm{I} 2625392)\end{array}$ & $\mathrm{KCl}$ & - & 22 & Yes\# & Wanner 2003 [24] \\
\hline & 9 & Joseph [79] & The Cochrane Library & $\mathrm{KCl}(\mathrm{No} \mathrm{ID})$ & $\mathrm{KCl}$ & - & 24 & Yes\# & Joseph 2000 [19] \\
\hline & 10 & Adams [26] & NRR & Unclear & No reply & Unclear & Unclear & No & No \\
\hline & II & Fryer [27] & ClinicalTrials.gov & Unclear & No reply & 120 & Unclear & No & No \\
\hline & 12 & $\begin{array}{c}\text { McCarthy } \\
{[28]}\end{array}$ & NRR & Unclear & Author ** & 160 & Unclear & No & No \\
\hline & 13 & Walker [29] & NRR & Unclear & No reply & 48 & Unclear & No & No \\
\hline Subtotal & 13 & & & & & 328 & 473 & & \\
\hline \multicolumn{10}{|l|}{ Discontinued } \\
\hline & 14 & No abstract & - & $\begin{array}{l}\mathrm{KCl}(\mathrm{PMID} \\
\mathrm{I} 2142596)\end{array}$ & $\mathrm{KCl}$ & - & 22 & Yes\# & $\begin{array}{l}\text { Interim results: Ford } \\
2002 \text { [30] }\end{array}$ \\
\hline & 15 & No abstract & $\mathrm{KCl}$ & $\begin{array}{c}\mathrm{KCl}(\mathrm{VAC} 200 \mathrm{I}- \\
02)\end{array}$ & $\mathrm{KCl}$ & 214 & - & No & No \\
\hline & 16 & Bayer [3I] & WUWHS 2004 & $\begin{array}{c}\mathrm{KCl}(\mathrm{VAC} \\
09)\end{array}$ & $\mathrm{KCl}$ & 116 & - & No & No \\
\hline & 17 & Greer [33] & The Cochrane Library & KCl (No ID) & $\mathrm{KCl}$ & t† 80 & - & No & No \\
\hline & 18 & Orgill [34] & WUWHS 2004 & $\begin{array}{c}\mathrm{KCl}(\mathrm{VAC} 2002- \\
10)\end{array}$ & $\mathrm{KCl}$ & 116 & - & No & No \\
\hline & 19 & Stannard [35] & WUWHS 2004 & $\mathrm{KCl}$ (VAC 200I- & $\mathrm{KCl}$; author & 300 & - & No & No \\
\hline
\end{tabular}


Table 4: Search results for randomised controlled trials on negative pressure wound therapy* (Status: December 2006) (Continued)

Subtotal 826 22

Ongoing

20 Armstrong WUWHS 2004

$21 \quad$ McCarthy

ClinicalTrials.gov

$\mathrm{KCl}(\mathrm{VAC} 200 \mathrm{I}-$

$\mathrm{KCl}$; author

08)

WUWHS 2004

Unclear

No reply

206

06

30

$-$

No

$\mathrm{KCl}$

$00)$

23 Niezgoda [39] WUWHS 2004

$\mathrm{KCl}$ (VAC 200I-

0 I)

24 Stannard [40]

WUWHS 2004

$\mathrm{KCl}$ (VAC 200I-

04)

25 Stannard [4I] WUWHS 2004

KCI (VAC 200I-

05)

$$
\mathrm{KCl}
$$

$\mathrm{KCl}$; author

214

$\mathrm{KCl}$; author

258
348

1106

-

(44)

(88)

(8)

$\begin{array}{cc}- & \text { No } \\ - & \text { No } \\ - & \text { No } \\ - & \text { No } \\ \text { Yestl } & \begin{array}{c}\text { Interim results: } \\ \text { Stannard 2006 [80] } \\ \text { Interim results: } \\ \text { Stannard 2006 [80] }\end{array}\end{array}$

Unclear

$\begin{array}{rc}26 & \text { Foo [42] } \\ 27 & \text { Gupta [43] } \\ 28 & \text { Lantis [44] } \\ 3 & \end{array}$

$\begin{array}{cc}\text { WUWHS 2004 } & \text { Unclear } \\ \text { WHS 2001 } & \text { Unclear } \\ \text { WUWHS 2004 } & \text { Unclear }\end{array}$

No reply
No reply
No reply

Unclear

(8)

Total (all

28

Unclear

No reply

$\begin{array}{llll}\text { Unclear } & - & \text { No } & \text { No } \\ \text { Unclear } & - & \text { No } & \text { No } \\ \text { Unclear } & - & \text { No } & \text { No } \\ \text { Unclear } & & & \end{array}$

trials)

* Not classified by IQWiG as an RCT on NPWT (in contrast to other systematic reviews): McCallon 2000 [69]; Genecov 1998 [70]; Buttenschoen 200। [8I]; Jeschke 2004 [82].

The PubMed ID number, when available, is used by $\mathrm{KCl}$ as the study ID for published RCTs.

Number of patients planned in unpublished and ongoing studies as stated in abstracts and personal communications.

$\S$ Number of patients analysed in published studies.

Full-text publication of interim or final results (any study status).

II Results considered in the IQWiG rapid report.

\# Results considered in the IQWiG systematic review.

** The author replied to IQWiG's first request for information and stated that the trial was ongoing. However, according to information provided by the NRR (accessed on 04 Feb 2007), this trial has since been completed. A second query was not answered.

t† According to $\mathrm{KCl}, \mathrm{I} 6$ patients were enrolled in this trial.

ID: Identification code; NRR: National Research Register; PMID: PubMed Identification Code; VLU: venous leg ulcer; WHS: Wound Healing Society; WUWHS: World Union of Wound Healing Societies. 
Search in bibliographic databases in

May 2005

MEDLINE ( $\mathrm{N}=1138)$

EMBASE $(\mathrm{N}=942)$

CINAHL $(\mathrm{N}=129)$

The Cochrane Library $(\mathrm{N}=303)$

Total citations: $\mathrm{N}=2512$

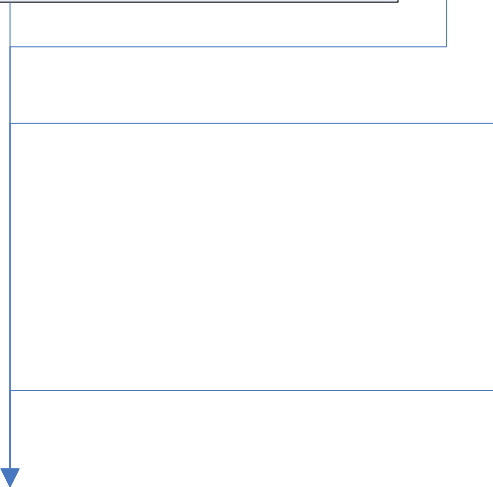

Citations including duplicates $\mathrm{N}=3634$

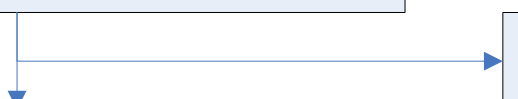

Citations excluding duplicates: $\mathrm{N}=2675$

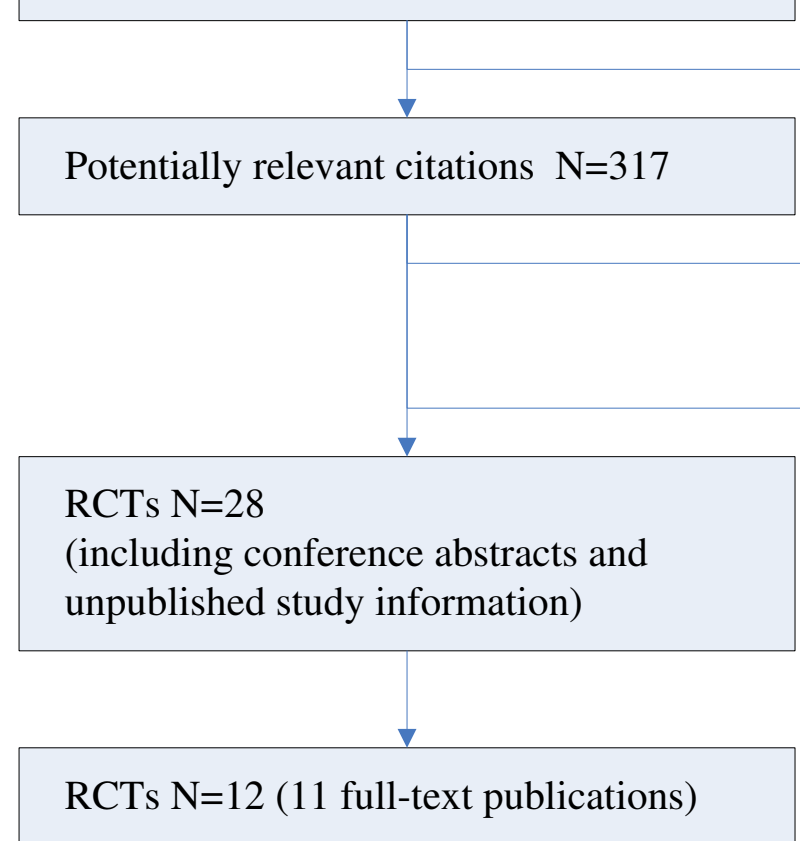

\section{Figure I}

Flow chart of the literature search for RCTs.
Exclusion: Duplicates N=959

Inclusion: Citations provided in 29 expert statements to the German Federal Joint Committee $\mathrm{N}=851$

Inclusion: Additional citations from congress reports, study registries, systematic reviews, manufacturers, and authors $\mathrm{N}=20$

Inclusion: Citations retrieved from bibliographic databases in the search update (December 2006) $\mathrm{N}=251$

Exclusion: Not relevant $\mathrm{N}=2323$

Exclusion: No RCT N=279

Exclusion: Systematic reviews $\mathrm{N}=10$ 
Table 5: Sources of conference abstracts and unpublished study information on 28 RCTs: databases, trial registers, and systematic reviews

\begin{tabular}{|c|c|c|c|c|c|c|c|c|c|}
\hline \multirow[t]{2}{*}{ No } & \multirow{2}{*}{$\begin{array}{l}\text { Abstracts or } \\
\text { other } \\
\text { sources }\end{array}$} & \multicolumn{2}{|c|}{ Bibliographic databases } & \multicolumn{2}{|c|}{ Trial registers } & \multicolumn{4}{|c|}{ Systematic reviews } \\
\hline & & $\begin{array}{l}\text { MEDLINE, } \\
\text { EMBASE or } \\
\text { CINAHL }\end{array}$ & $\begin{array}{l}\text { Cochrane } \\
\text { Clinical } \\
\text { Trials }\end{array}$ & CT.gov & NRR & $\begin{array}{c}\text { Evans } \\
2001 \text { [83] }\end{array}$ & $\begin{array}{l}\text { Samson } \\
2004 \text { [9] }\end{array}$ & $\begin{array}{c}\text { Pham } \\
2006[68]\end{array}$ & $\begin{array}{l}\text { Fisher } 2003 \text { [84]; } \\
\text { Higgins } 2003 \text { [85]; } \\
\text { Costa } 2005 \text { [67]; } \\
\text { OHTAC } 2006 \text { [10] }\end{array}$ \\
\hline 1 & Vuerstaek [74] & - & + & - & - & - & + & - & - \\
\hline 2 & No abstract* & - & - & - & - & - & - & - & - \\
\hline 3 & Obdeijn [75] & - & + & - & - & - & + & - & - \\
\hline 4 & Payne [76] & - & + & - & - & - & + & - & - \\
\hline 5 & Moues [77] & - & + & - & - & - & + & - & - \\
\hline 6 & Heath [78] & - & + & - & - & + & - & + & - \\
\hline 7 & No abstract ${ }^{\dagger}$ & - & - & - & - & - & - & - & - \\
\hline 8 & No abstract $\ddagger$ & - & - & - & - & - & - & - & - \\
\hline 9 & Joseph [79] & - & + & - & - & - & - & - & - \\
\hline 10 & Adams [26] & - & - & - & + & - & - & + & - \\
\hline 11 & Fryer [27] & - & - & + & - & - & - & + & - \\
\hline 12 & McCarthy [28] & - & - & - & + & - & - & + & - \\
\hline 13 & Walker [29] & - & - & - & + & - & - & + & - \\
\hline 14 & No abstract $\S$ & - & - & - & - & - & - & - & - \\
\hline 15 & No abstractll & - & - & - & - & - & + & - & - \\
\hline 16 & Bayer [3I] & - & + & - & - & - & + & - & - \\
\hline 17 & Greer [33] & - & + & - & - & + & - & + & - \\
\hline 18 & Orgill [34] & - & + & - & - & - & + & - & - \\
\hline 19 & Stannard [35] & - & + & - & - & - & + & - & - \\
\hline 20 & $\begin{array}{l}\text { Armstrong } \\
{[36]}\end{array}$ & - & + & - & - & - & + & - & - \\
\hline 21 & McCarthy [37] & - & - & + & - & - & - & - & - \\
\hline 22 & Molnar [38] & - & + & - & - & - & + & - & - \\
\hline 23 & Niezgoda [39] & - & + & - & - & - & + & - & - \\
\hline 24 & Stannard [40] & - & + & - & - & - & + & - & - \\
\hline 25 & Stannard [4I] & - & + & - & - & - & + & - & - \\
\hline 26 & Foo [42] & - & + & - & - & - & + & - & - \\
\hline 27 & Gupta [43] & - & + & - & - & - & - & - & - \\
\hline \multirow[t]{2}{*}{28} & Lantis [44] & - & + & - & - & - & + & - & - \\
\hline & & 0 & 18 & 2 & 3 & 2 & 15 & 6 & 0 \\
\hline
\end{tabular}

\footnotetext{
* No preceding abstract was identified concerning the full-text article by Llanos 2006 (Tables 7-9).

$\dagger$ No preceding abstract was identified, but information by the manufacturer $\mathrm{KCl}$ was provided concerning the full-text article by Eginton 2003 (Tables 7-9).

$\ddagger$ No preceding abstract was identified, but information by the manufacturer $\mathrm{KCl}$ was provided concerning the full-text article by Wanner 2003 (Tables 7-9).

$\S$ No preceding abstract was identified, but information by the manufacturer $\mathrm{KCl}$ was provided concerning the full-text article by Ford 2002 (Tables 7-9).

|| No preceding abstract was identified, but information by the manufacturer $\mathrm{KCl}$ was provided concerning this discontinued study KCl ID VAC 2001-02.

CT.gov: ClinicalTrials.gov (United States); NRR: National Research Register (United Kingdom National Health System); RCT: randomised controlled trial
}

RCTs (1106 patients; 40\%) were ongoing [36-41], and the status of 3 RCTs was unclear [42-44] (Table 3). According to $\mathrm{KCI}$, the main reasons for discontinuation were slow enrolment, high attrition rates, changes in clinical practice, or design flaws [6].
Full-text publications were available on 30\% of the 1649 patients in the 19 completed or discontinued RCTs (495 analysed patients in 10 published RCTs vs. 1154 planned patients in 9 unpublished RCTs). Of the 14 conference abstracts on these 19 trials, 6 abstracts (43\%) were later published as full-text articles. 
Table 6: Sources of conference abstracts and unpublished study information on 28 RCTs: manufacturers, authors, congress proceedings, and authorities

\begin{tabular}{|c|c|c|c|c|c|c|c|c|}
\hline \multirow[t]{2}{*}{ No } & \multirow{2}{*}{$\begin{array}{l}\text { Abstracts or } \\
\text { other sources }\end{array}$} & \multicolumn{2}{|c|}{ Manufacturers } & \multirow[t]{2}{*}{ Authors* } & \multicolumn{2}{|c|}{ Congress proceedings* } & \multicolumn{2}{|c|}{ Authorities* } \\
\hline & & $\begin{array}{l}\text { Kinetic Concepts, } \\
\text { Inc. }\end{array}$ & Blue Sky Medical & & $\begin{array}{c}\text { WUWHS } 2004 \\
{[45]}\end{array}$ & Others & Experts $2003 \dagger$ & Others \\
\hline 1 & Vuerstaek [74] & + & - & - & + & - & - & - \\
\hline 2 & No abstract $\ddagger$ & - & - & - & - & - & - & - \\
\hline 3 & Obdeijn [75] & + & - & - & + & - & + & - \\
\hline 4 & Payne [76] & + & - & + & + & - & - & - \\
\hline 5 & Moues [77] & + & - & - & + & - & + & - \\
\hline 6 & Heath [78] & + & - & + & - & - & - & - \\
\hline 7 & No abstract $\S$ & + & - & - & - & - & - & - \\
\hline 8 & No abstractll & + & - & - & - & - & - & - \\
\hline 9 & Joseph [79] & + & - & - & - & - & + & - \\
\hline 10 & Adams [26] & - & - & - & - & - & - & - \\
\hline 11 & Fryer [27] & - & - & - & - & - & - & - \\
\hline 12 & McCarthy [28] & - & - & + & - & - & - & - \\
\hline 13 & Walker [29] & - & - & - & - & - & - & - \\
\hline 14 & No abstract & + & - & - & - & - & - & - \\
\hline 15 & No abstract $\#$ & + & - & - & - & - & - & - \\
\hline 16 & Bayer [3I] & + & - & - & + & - & - & - \\
\hline 17 & Greer [33] & + & - & - & - & - & + & - \\
\hline 18 & Orgill [34] & + & - & - & + & - & - & - \\
\hline 19 & Stannard [35] & + & - & - & + & - & - & - \\
\hline 20 & Armstrong [36] & + & - & + & + & - & - & - \\
\hline 21 & McCarthy [37] & - & - & - & - & - & - & - \\
\hline 22 & Molnar [38] & + & - & - & + & - & - & - \\
\hline 23 & Niezgoda [39] & + & - & - & + & + & - & - \\
\hline 24 & Stannard [40] & + & - & - & + & - & - & - \\
\hline 25 & Stannard [4I] & + & - & - & + & - & - & - \\
\hline 26 & Foo [42] & - & - & - & + & - & - & - \\
\hline 27 & Gupta [43] & - & - & - & - & + & - & - \\
\hline \multirow[t]{2}{*}{28} & Lantis [44] & - & - & - & + & - & + & - \\
\hline & & 19 & 0 & 4 & 14 & 2 & 5 & 0 \\
\hline
\end{tabular}

\footnotetext{
* See Table I.
}

† Citations submitted by experts to the German Federal Joint Committee.

$¥$ No preceding abstract was identified concerning the full-text article by Llanos 2006 (Tables 7-9).

$\S$ No preceding abstract was identified, but information by the manufacturer $\mathrm{KCl}$ was provided concerning the full-text article by Eginton 2003 (Tables 7-9).

|| No preceding abstract was identified, but information by the manufacturer $\mathrm{KCl}$ was provided concerning the full-text article by Wanner 2003 (Tables 7-9).

INo preceding abstract was identified, but information by the manufacturer $\mathrm{KCl}$ was provided concerning the full-text article by Ford 2002 (Tables 7-9).

\# No preceding abstract was identified, but information by the manufacturer $\mathrm{KCl}$ was provided concerning this discontinued study $\mathrm{KCl}$ ID VAC $200 \mathrm{I}-02$.

RCT: randomised controlled trial; WUWHS: World Union of Wound Healing Societies

\section{Response behaviour of manufacturers and authors}

A total of 17 authors and 2 manufacturers were contacted, of whom 10 (59\%) and 2 (100\%) responded respectively. KCI readily provided information on study and publication status and on methodological issues. BSM did not sponsor relevant RCTs and provided information only on case reports.
One author provided information on an RCT that had been classified as "ongoing", and stated that this trial had recently been completed and the manuscript submitted to The Lancet [22]. As this trial included 162 analysed patients and was the largest RCT on NPWT conducted so far, IQWiG postponed the publication of its review by 2 months until the final results of this RCT were available, in order to include the results in the body of evidence. 
Table 7: Sources of I I published full-text articles on I2 RCTs: databases and trial registers

\begin{tabular}{|c|c|c|c|c|c|c|c|}
\hline \multirow[t]{2}{*}{ No } & \multirow[t]{2}{*}{ Full-text articles } & \multicolumn{4}{|c|}{ Bibliographic databases } & \multicolumn{2}{|c|}{ Trial registers } \\
\hline & & MEDLINE & EMBASE & Cochrane Clinical Trials & CINAHL & CT.gov & NRR \\
\hline I & Braakenburg 2006 [2I] & + & + & + & - & - & - \\
\hline 2 & Stannard $2006[80]^{*}$ & + & + & + & - & - & - \\
\hline 3 & Vuerstaek 2006 [23] & + & - & + & - & + & - \\
\hline 4 & Llanos 2006 [25] & + & + & + & - & - & - \\
\hline 5 & Armstrong 2005 [22] & + & + & + & + & + & - \\
\hline 6 & Moisidis 2004 [18] & + & + & + & - & - & - \\
\hline 7 & Moues 2004 [20] & + & + & + & + & - & - \\
\hline 8 & Eginton 2003 [17] & + & + & + & - & - & - \\
\hline 9 & Wanner 2003 [24] & + & + & + & - & - & - \\
\hline 10 & Ford $2002[30]$ & + & + & + & - & - & - \\
\hline \multirow[t]{2}{*}{11} & Joseph 2000 [19] & - & - & + & + & - & - \\
\hline & & 10 & 9 & I I & 3 & 2 & 0 \\
\hline
\end{tabular}

* Stannard 2006 [80]: one article presenting preliminary results of 2 ongoing RCTs [40,4I].

CT.gov: ClinicalTrials.gov (United States); NRR: National Research Register (United Kingdom National Health System); RCT: randomised controlled trial

\section{Literature sources}

The sources of the conference abstracts and unpublished study information referring to 28 RCTs on NPWT are shown in Tables 5, 6: most information was obtained from KCI (19), followed by Cochrane (18), a systematic review (15) [9], and congress proceedings (14) [45].
The sources of all 11 published full-text articles (referring to 12 completed, discontinued or ongoing RCTs) are shown in Tables 7, 8, 9. All 11 articles were obtained from the Cochrane Library followed by KCI (10), MEDLINE (10), and EMBASE (9).

Table 8: Sources of II published full-text articles on I 2 RCTs: systematic reviews

\begin{tabular}{|c|c|c|c|c|c|c|c|c|}
\hline \multirow[t]{2}{*}{ No } & \multirow{2}{*}{$\begin{array}{l}\text { Full-text } \\
\text { articles }\end{array}$} & \multicolumn{7}{|c|}{ Systematic reviews } \\
\hline & & $\begin{array}{c}\text { Evans 200I } \\
{[83]}\end{array}$ & $\begin{array}{c}\text { Fisher } 2003 \\
{[84]}\end{array}$ & $\begin{array}{c}\text { Higgins } 2003 \\
{[85]}\end{array}$ & $\begin{array}{c}\text { Samson } 2004 \\
{[9]}\end{array}$ & $\begin{array}{c}\text { Costa } 2005 \\
{[67]}\end{array}$ & $\begin{array}{c}\text { Pham } 2006 \\
{[68]}\end{array}$ & $\begin{array}{c}\text { OHTAC } 2006 \\
{[10]}\end{array}$ \\
\hline 1 & $\begin{array}{l}\text { Braakenburg } \\
2006[2 I]\end{array}$ & - & - & - & - & - & - & - \\
\hline 2 & $\begin{array}{l}\text { Stannard } 2006 \\
{[80]^{*}}\end{array}$ & - & - & - & - & - & - & - \\
\hline 3 & $\begin{array}{l}\text { Vuerstaek } 2006 \\
\text { [23] }\end{array}$ & - & - & - & - & - & - & - \\
\hline 4 & Llanos 2006 [25] & - & - & - & - & - & - & - \\
\hline 5 & $\begin{array}{l}\text { Armstrong } 2005 \\
{[22]}\end{array}$ & - & - & - & - & - & - & + \\
\hline 6 & $\begin{array}{l}\text { Moisidis } 2004 \\
\text { [18] }\end{array}$ & - & - & - & - & + & + & + \\
\hline 7 & Moues 2004 [20] & - & - & - & + & + & + & + \\
\hline 8 & $\begin{array}{l}\text { Eginton } 2003 \\
\text { [17] }\end{array}$ & - & - & - & + & + & + & - \\
\hline 9 & $\begin{array}{l}\text { Wanner } 2003 \\
\text { [24] }\end{array}$ & - & - & + & + & + & + & + \\
\hline 10 & Ford 2002 [30] & - & + & + & + & + & + & + \\
\hline \multirow[t]{2}{*}{11} & Joseph 2000 [19] & + & + & + & + & + & + & + \\
\hline & & I & 2 & 3 & 5 & 6 & 6 & 6 \\
\hline
\end{tabular}

* Stannard 2006 [80]: one article presenting preliminary results of 2 ongoing RCTs [40,4I].

RCT: randomised controlled trial 
Table 9: Sources of I I published full-text articles on I 2 RCTs: manufacturers, authors, congress proceedings, and authorities

\begin{tabular}{|c|c|c|c|c|c|c|c|}
\hline \multirow[b]{2}{*}{ No } & \multirow[b]{2}{*}{ Full-text articles } & \multicolumn{2}{|c|}{ Manufacturers } & \multirow[t]{2}{*}{ Authors* } & \multirow{2}{*}{$\begin{array}{c}\text { Congress } \\
\text { proceedings* }\end{array}$} & \multicolumn{2}{|c|}{ Authorities* } \\
\hline & & $\begin{array}{l}\text { Kinetic Concepts, } \\
\text { Inc. }\end{array}$ & Blue Sky Medical & & & Experts $2003 \dagger$ & Others \\
\hline I & Braakenburg 2006 [2I] & + & - & - & - & - & - \\
\hline 2 & Stannard $2006[80]^{\ddagger}$ & + & - & - & - & - & - \\
\hline 3 & Vuerstaek 2006 [23] & + & - & - & - & - & - \\
\hline 4 & Llanos 2006 [25] & - & - & - & - & - & - \\
\hline 5 & Armstrong 2005 [22] & + & - & - & - & - & - \\
\hline 6 & Moisidis 2004 [18] & + & - & - & - & - & - \\
\hline 7 & Moues 2004 [20] & + & - & - & - & - & - \\
\hline 8 & Eginton 2003 [17] & + & - & - & - & + & - \\
\hline 9 & Wanner 2003 [24] & + & - & - & - & + & - \\
\hline 10 & Ford 2002 [30] & + & - & - & - & + & - \\
\hline \multirow[t]{2}{*}{11} & Joseph 2000 [19] & + & - & - & - & + & - \\
\hline & & 10 & 0 & 0 & 0 & 4 & 0 \\
\hline
\end{tabular}

* See Table I.

† Citations submitted by experts to the German Federal Joint Committee.

$\ddagger$ Stannard 2006 [80]: one article presenting preliminary results of 2 ongoing RCTs [40,4I].

RCT: randomised controlled trial

\section{Summary of quality assessment and outcomes}

\section{Published trials}

The overall methodological quality of the 12 published RCTs included in the IQWiG systematic review and rapid report was poor. Methodological flaws included the lack of blinding, the lack of intention-to-treat analyses, inadequate allocation concealment, the unclear definition of primary outcomes, and high drop-out rates. Significant differences in favour of NPWT for wound healing parameters, such as time to wound closure or the incidence of wound closure, were shown in 3 of 7 RCTs analysing these outcomes. Data on other patient-relevant outcomes, such as reoperation rates or pain scores, were scarce or not interpretable.

\section{Unpublished trials}

Because of insufficient available information on the 9 completed or discontinued unpublished RCTs, an assessment of their quality could not be conducted. Regarding the 5 discontinued industry-sponsored trials, as stated, the reasons for discontinuation were provided by KCI, which also reported the number of enrolled patients for one trial [33]. However, there was no response to a further request from IQWIG asking for more detailed information on all discontinued trials, such as the number of enrolled patients and other results data. Regarding the 4 completed RCTs with unclear sponsorship, the authors of publications did not respond to IQWiG's request to provide results data (Table 4). Consequently, the results of unpublished RCTs could not be considered in the review.

\section{Industrial sponsorship}

The manufacturer KCI sponsored the majority (19/28) of RCTs $(68 \%$ of trials referring to $85 \%$ of patients planned or analysed). The sponsorship of the remaining trials was unclear.

\section{Discussion}

The main objective of this paper was to identify unpublished RCTs on NPWT within the framework of a systematic review. An RCT was classified as "unpublished" if no full-text paper on final study results (completed trials) or interim results (discontinued trials) was available.

An extensive search strategy was employed that included handsearching of retrievals, as well as contacting manufacturers and authors of publications. The sensitivity of bibliographic database searching for RCTs is reported to be unsatisfactory [46], and multi-source searching has been recommended to retrieve all available RCTs [47]. In this context, the usefulness of handsearching and contacting experts has been demonstrated $[48,49]$. The extensive search strategy was also useful to detect ongoing RCTs, of which one [22] was subsequently added to the evidence base.

A total of 28 RCTs referring to at least 2755 patients were identified. The publications on completed or discontinued RCTs reported data on less than a third of patients planned or analysed. Less than half of the conference abstracts on these trials were later published as full-text publications; similar results were found in a review by Scherer et al, who found a full-text publication rate for 
abstracts describing RCTs or controlled clinical trials of about $60 \%$ [50].

On the basis of results of published data alone, the IQWiG review and rapid report concluded that although there was some indication that NPWT may improve wound healing, the body of evidence available was insufficient to clearly prove an additional clinical benefit of NPWT. This finding is in line with findings from previous reviews.

Regarding the discontinued unpublished trials, according to the manufacturer the main reason for discontinuation was insufficient patient numbers. No results data for these trials were available. Regarding the completed unpublished trials, the authors of conference abstracts did not respond to IQWiG's request for information. Therefore, unpublished results data could not be considered in the IQWiG review and consequently the impact of unpublished data on the conclusions of reviews based on published data could not be assessed.

\section{Publication bias caused by unpublished data}

Previous research has shown that inclusion of unpublished data in systematic reviews may affect the review's prior conclusions $[12,13]$. The most prominent example in recent years has been the evidence on serotonin reuptake inhibitors (SSRIs) in paediatric depression: a systematic review of published versus unpublished data showed that whereas published trials indicated a favourable risk-benefit profile, the addition of unpublished data reversed this profile for some SSRIs [11].

Klassen 2002 assessed the proportion of RCTs presented at a major paediatric meeting that were subsequently published as full publications; about $60 \%$ of abstracts were subsequently published, and RCTs were more likely to be published if they favoured the new therapy. The author concluded that "publication bias is a serious threat to assessing the effectiveness of interventions in child health" and called for a registry of RCTs in children so that the totality of evidence could be assessed [51].

As unpublished results data on NPWT were not available, no statement can be made on the impact of unpublished data on the validity of published evidence. However, if one assumes a "worst case scenario" (all unpublished trials show results in favour of conventional therapy), the current conclusion would no longer hold that there is some indication of a potential advantage of NPWT over conventional therapy.

Besides possible problems of statistical evaluation due to insufficient sample sizes, the reasons for the existence of unpublished RCTs on NPWT remain unclear and one can only speculate. For example, it may be possible that some of these RCTs remained unpublished because they were of poor quality or produced negative results. Previous research has shown that studies with statistically significant or positive results are more likely to be published than those with non-significant or negative results, and are also published earlier [52-54]. These studies also lead to a greater number of publications, and are more likely to be published in high impact factor journals [55]. As for discontinued trials, even if small sample sizes make statistical evaluation difficult, in our opinion the full datasets of such trials should be publicly accessible to ensure that the evidence base is complete.

The inclusion of unpublished data in systematic reviews is controversial. Although less than a third of meta-analyses include unpublished data, nearly $80 \%$ of meta-analysts and methodologists believe that unpublished data should definitely or probably be included in systematic reviews; in contrast, less than half of journal editors agree [56]. The identification of unpublished data has been recommended to minimise the risk of bias in systematic reviews [12], but there are variations in policy regarding their inclusion [57]. This may be explained by incomplete or inaccurate reporting and the subsequent difficulties in assessing the methodological quality of trials $[57,58]$.

In general, the quality of reporting of systematic reviews on both drug and non-drug interventions is inconsistent [59]. Moreover, systematic reviews on non-drug interventions (e.g. surgical techniques) face specific problems, such as the limited quality and quantity of RCTs [60]. Searching for unpublished data and conducting sensitivity analyses to assess their impact is recommended for topics with little evidence and new or evolving interventions [58]. In their commentary on the problem of conducting systematic reviews of new health technologies, Moher and Schachter concluded that the inclusion of unpublished data was of "paramount importance" in assessing the usefulness of an intervention [61].

\section{The role of industrial sponsorship}

Approximately two thirds of the RCTs on NPWT were sponsored by one manufacturer (KCI). Industrial sponsorship of clinical trials is common. The percentage of industry-sponsored clinical trials has increased to over $60 \%$ in recent years and the number of industry employees named as co-authors of clinical trial publications is rising [62]. However, the role of industrial sponsorship in clinical research is controversial. Industry sponsorship is discussed as being associated with the following factors: selection of inappropriate comparators, selective reporting of more favourable per protocol analyses, pro-industry conclusions, as well as restrictions on publication and data sharing [63-65]. A comparison of Cochrane reviews versus industry-supported meta-analyses showed that the 
latter came to more favourable conclusions on the same drugs [66]. The available data on NPWT are insufficient to make definite conclusions about the impact of industrial sponsorship in this field.

\section{Differences in primary study selection}

Five systematic reviews on NPWT (including the IQWiG review) published in December 2004 or later showed considerable differences in the selection of primary studies published between 1994 and 2004 [6,9,10,67,68]. There was agreement between reviews regarding the selection of 4 RCTs $[19,20,24,30]$. One further study [17] classified by IQWiG as an eligible RCT was not classified accordingly (or identified) by 2 other reviews [10,67]. A study [69] classified as an eligible RCT by 2 reviews $[9,68]$ was classified as non-randomised by IQWiG, and one study [70] classified as an eligible RCT by one other review [68] was also classified as non-randomised by IQWiG. These findings indicate that in systematic reviews on NPWT, search strategies, inclusion criteria, and classification of primary studies are not applied in a standardised manner.

\section{Conclusion}

Multi-source comprehensive searches identify unpublished clinical trial data. However, lack of access to unpublished study results data raises doubts about the completeness of the evidence base on NPWT. The implementation of regulations such as prospective mandatory trial registration and the obligation to publish all results is needed to ensure that independent researchers have access to all outcomes of completed or discontinued clinical trials.

\section{Competing interests}

The author(s) declare that they have no competing interests.

\section{Authors' contributions}

StL initiated the study. FP coordinated the study and conducted the literature search. StL, FP, and StS screened and analysed the retrievals. NM and FP drafted the manuscript. All authors interpreted the data and made an intellectual contribution to the manuscript. All authors reviewed and approved the final version.

\section{Acknowledgements}

IQWiG is an independent non-profit and non-government institute that evaluates the quality and efficiency of health care services in Germany. All work on this study was supported by IQWiG within the framework of a systematic review on NPWT performed by IQWiG. Three authors (FP, NM, StL) are IQWiG full-time employees; one author (StS) works as an external expert for IQWiG.

\section{References}

I. Graham ID, Harrison MB, Nelson EA, Lorimer K, Fisher A: Prevalence of lower-limb ulceration: a systematic review of prevalence studies. Adv Skin Wound Care 2003, 16:305-316.
2. Kaltenthaler E, Whitfield MD, Walters SJ, Akehurst RL, Paisley S: UK, USA and Canada: how do their pressure ulcer prevalence and incidence data compare? J Wound Care 200I, 10:530-535.

3. Wu S, Armstrong DG: Risk assessment of the diabetic foot and wound. Int Wound J 2005, 2: 17-24.

4. Persoon A, Heinen MM, van V, de Rooij MJ, van de Kerkhof PC, van AT: Leg ulcers: a review of their impact on daily life. J Clin Nurs 2004, 13:34I-354.

5. Argenta LC, Morykwas MJ: Vacuum-assisted closure: a new method for wound control and treatment: clinical experience. Ann Plast Surg 1997, 38:563-577.

6. Negative pressure wound therapy. Final report (German version) 2006 [http://www.iqwig.de/download/N04-

03 Abschlussbericht Vakuumversiegelungstherapie zur Behandlung von Wunden..pdf]. Cologne, Germany, Institute for Quality and Efficiency in Health Care (IQWiG)

7. Gregor S, Maegele M, Sauerland S, Krahn J, Peinemann F, Lange S: Negative pressure wound therapy: A vacuum of evidence? Arch Surg 2008, 143:189-196.

8. Negative pressure wound therapy. Rapid report (German version) 2007 [http://www.iqwig.de/download/N06-

02 Rapid Report Vakuumversiegelungstherapie von Wunden..pdf]. Cologne, Germany, Institute for Quality and Efficiency in Health Care (IQWiG)

9. Samson DJ, Lefevre F, Aronson N: Wound healing technologies: Lowlevel laser and vacuum-assisted closure. 2004 [http://www.ahrq.gov/ clinic/epcsums/woundsum.htm]. Rockville, MD, USA, Agency for Healthcare Research and Quality (AHRQ)

10. Ontario Health Technology Advisory Committee (OHTAC): Negative pressure wound therapy: Update 2006:I-38 [http:/l www.health.gov.on.ca/english/providers/program/ohtac/tech/ reviews/pdf/rev npwt 070106.pdf]. Toronto, Ontario, Canada, Ministry of Health and Long-Term Care (MOHLTC) Medical Advisory Secretariat (MAS)

II. Whittington CJ, Kendall T, Fonagy P, Cottrell D, Cotgrove A, Boddington E: Selective serotonin reuptake inhibitors in childhood depression: systematic review of published versus unpublished data. Lancet 2004, 363: I34 I-1345.

12. Hopewell S, McDonald S, Clarke M, Egger M: Grey literature in metaanalyses of randomized trials of health care interventions. Cochrane Database of Methodology Reviews 2006, Issue 2. Art. 2006 [http:// www.mrw.interscience.wiley.com/cochrane/clsysrev/articles/ MR0000 I0/pdf fs.html]. West Sussex, United Kingdom, John Wiley \& Sons, Ltd

13. McAuley L, Pham B, Tugwell P, Moher D: Does the inclusion of grey literature influence estimates of intervention effectiveness reported in meta-analyses? Lancet 2000, 356: I228- 123 I.

14. Appendix 5b: Highly sensitive search strategies for identifying reports of randomized controlled trials in MEDLINE. Cochrane Handbook for Systematic Reviews of Interventions 4.2.6 [updated September 2006] The Cochrane Library, Issue 4, 2006 2006:182-I86 [http://www.cochrane.org/resources/ handbook/Handbook4.2.6Sep2006.pdf]. Chichester, UK, John Wiley \& Sons, Ltd

15. Methods Version 2.02007 [http://www.iqwig.de/download/ Methods a second Version Produced by the Institutes Steering Committee.pdf]. Cologne, Germany, Institute for Quality and Efficiency in Health Care (IQWiG)

16. Jüni P, Altman DG, Egger M: Assessing the quality of randomised controlled trials. In Systematic reviews in health care - meta-analysis in context Edited by: Egger M, Davey Smith G and Altman DG. London, UK, BMJ Publishing Group; 2001:87-108.

17. Eginton MT, Brown KR, Seabrook GR, Towne JB, Cambria RA: A prospective randomized evaluation of negative-pressure wound dressings for diabetic foot wounds. Ann Vasc Surg 2003, 17:645-649.

18. Moisidis E, Heath T, Boorer C, Ho K, Deva AK: A prospective, blinded, randomized, controlled clinical trial of topical negative pressure use in skin grafting. In Plast Reconstr Surg Volume 1/4. United States; 2004:917-922.

19. Joseph E, Hamori CA, Bergman S, Roaf E, Swann NF, Anastasi GW: A prospective randomized trial of vacuum-assisted closure versus standard therapy of chronic nonhealing wounds. Wounds A Compendium of Clinical Research and Practice 2000, 12:60-67.

20. Moues CM, Vos MC, van den Bemd GJ, Stijnen T, Hovius SE: Bacterial load in relation to vacuum-assisted closure wound ther- 
apy: a prospective randomized trial. In Wound Repair Regen Volume 12. United States; 2004:II-17.

21. Braakenburg A, Obdeijn MC, Feitz R, van Rooij IALM, van Griethuysen AJ, Klinkenbij JHG: The clinical efficacy and cost effectiveness of the vacuum-assisted closure technique in the management of acute and chronic wounds: a randomized controlled trial. In Plast Reconstr Surg Volume / /8. United States; 2006:390-397.

22. Armstrong DG, Lavery LA: Negative pressure wound therapy after partial diabetic foot amputation: a multicentre, randomised controlled trial. Lancet 2005, 366: 1704-1710.

23. Vuerstaek JD, Vainas T, Wuite J, Nelemans P, Neumann MH, Veraart JC: State-of-the-art treatment of chronic leg ulcers: A randomized controlled trial comparing vacuum-assisted closure (V.A.C.) with modern wound dressings. J Vasc Surg 2006, 44:1029-1037.

24. Wanner MB, Schwarzl F, Strub B, Zaech GA, Pierer G: Vacuumassisted wound closure for cheaper and more comfortable healing of pressure sores: a prospective study. Scand J Plast Reconstr Surg Hand Surg 2003, 37:28-33.

25. Llanos S, Danilla S, Barraza C, Armijo E, Pineros JL, Quintas M, Searle $S$, Calderon W: Effectiveness of negative pressure closure in the integration of split thickness skin grafts: a randomized, double-masked, controlled trial. Ann Surg 2006, 244:700-705.

26. Adams TST: The effect of topical negative pressure on donor site wound healing P003. 1999 [https://portal.nihr.ac.uk/Profiles/ NRR.aspx?Publication ID=N0245095365]. London, UK, National Research Register, Department of Health

27. Fryer J: Investigation of Subatmospheric Pressure Dressing on Pressure Ulcer Healing. $200 \mathrm{I}$ [http://clinicaltrials.gov/ct/showl NCT000I I53I? order=I]. Bethesda, Maryland, USA, ClinicalTrials.gov, National Institutes of Health (NIH)

28. McCarthy M: Vacuum Assisted Closure for the Management of Ischaemic Wounds in the Lower Limb: A Randomised Controlled Trial and In Vitro Studies. 2005 [https://por tal.nihr.ac.uk/Profiles/NRR.aspx?Publication ID=N0I23I38623]. London, UK, National Research Register, Department of Health

29. Walker P: A Randomised, Prospective Trial of Standard Wound Drainage Medinorm VS Constant Vacuum Drainage to Determine Whether There is any Effect on the Amount of Wound Exudate, Haemoglobin \& Wound Bruising. 1998 [https://portal.nihr.ac.uk/Profiles/

NRR.aspx?Publication ID=N0084029434]. London, UK, National Research Register, Department of Health

30. Ford CN, Reinhard ER, Yeh D, Syrek D, De Las MA, Bergman SB, Williams $S$, Hamori CA: Interim analysis of a prospective, randomized trial of vacuum-assisted closure versus the healthpoint system in the management of pressure ulcers. Ann Plast Surg 2002, 49:55-61.

31. Bayer L, Orgill DP: Has the wound VAC become the standard of care for the sternal wounds? Abstract DD004. Paris, France, Second World Union of Wound Healing Societies Meeting (08-13 July); 2004.

32. KCI ID: VAC 200I-02. Venous stasis ulcer. Personal communication from KCl to IQWiG. 2005.

33. Greer SE, Longaker MT, Margiotta M: Preliminary results from a multicenter, randomized, controlled study of the use of subatmospheric pressure dressing for pressure ulcer healing. Wound Repair Regen 1999, 7:Abstract A255..

34. Orgill DP, Bayer L: Preliminary results indicate VAC therapy facilitates faster closure of open abdominal wounds. Abstract DD0 I0. In Presented at the 2nd World Union of Wound Healing Societies Meeting 2004 (July 8-13), Paris, France Paris, France, Second World Union of Wound Healing Societies Meeting (08-13 July); 2004

35. Stannard JP, Volgas DA, Robinson J, Anglen JO, De Los Santos A: Topical negative pressure therapy for soft tissue management of open fractures: Preliminary results. Abstract EO IO. Paris, France, Second World Union of Wound Healing Societies Meeting (08-13 July); 2004.

36. Armstrong DG, Lavery LA, Frykberg RG, Andros G, Attinger CE, Boulten AJM: VAC therapy appears to heal complex DFU. Abstract HOI3. Paris, France, Second World Union of Wound Healing Societies Meeting (08-13 July); 2004.

37. McCarthy J: Study to Determine the Utility of Wound Vacuum Assisted Closure (VAC) Compared to Conventional
Saline Dressing Changes. 2005 [http://clinicaltrials.gov/ct/show/ NCT00121537!order=1]. Bethesda, Maryland, USA, ClinicalTrials.gov, National Institutes of Health (NIH)

38. Molnar JA, Heimbach DM, Tredgett EE, Mozingo DW: Prospective, randomized, controlled, multicenter trial applying subatmospheric pressure to acute hand burns: An interim report. Abstract D008. Paris, France, Second World Union of Wound Healing Societies Meeting (08-13 July); 2004.

39. Niezgoda JA: A Comparison of Vacuum Assisted Closure Therapy to Moist Wound Care in the Treatment of Pressure Ulcers: Preliminary Results of a Multicenter Trial. Abstract X00I. In Presented at the 2nd World Union of Wound Healing Societies Meeting 2004 (July 8-13), Paris, France Paris, France, Second World Union of Wound Healing Societies Meeting (08-13 July); 2004.

40. Stannard JP, Volgas DA, Robinson J, Anglen JO, De Los Santos A: Topical negative pressure therapy as a treatment for draining hematomas following surgical stabilization of skeletal trauma: preliminary results. Abstract E008. In Presented at the 2nd World Union of Wound Healing Societies Meeting 2004 (July 8-13), Paris, France Paris, France, Second World Union of Wound Healing Societies Meeting (08-13 July); 2004.

41. Stannard JP, Volgas DA, Robinson J, Anglen JO, De Los Santos A: Topical negative pressure therapy as an adjunct to soft tissue healing following open reduction and internal fixation of high risk fractures: preliminary results. Abstract E009. Paris, France, Second World Union of Wound Healing Societies Meeting (08-I 3 July); 2004.

42. Foo LSS, Chua BSY, Chia GT, Tan SB, Howe TS: Vacuum assisted closure vs moist gauze dressing in post-operative diabetic foot wounds: Early results from a randomised controlled trial. Abstract. In Presented at the 2nd World Union of Wound Healing Societies Meeting (July 8-I3), Paris, France Paris, France, Second World Union of Wound Healing Societies Meeting (08-13 July); 2004.

43. Gupta SC: Sterilizing chronic wounds with negative pressure therapy: the role of antibiotic irrigation. Abstract 127. Albuquerque, New Mexico, USA., II th Annual Meeting and Educational Symposuim, Wound Healing Society (I6-18 May); 200I.

44. Lantis JC, Gendics C: VAC therapy appears to facilitate STSG take when applied to venous leg ulcers. Abstract P036. In Presented at the 2nd World Union of Wound Healing Societies Meeting July 8-13), Paris, France Paris, France, Second World Union of Wound Healing Societies Meeting (08-13 July); 2004.

45. Abstracts of the 2nd World Union of Wound Healing Societies, Paris, France, July 8-13, 2004. Wound Repair Regen 2005, 13:A49-A87.

46. Dickersin K, Scherer R, Lefebvre C: Identifying relevant studies for systematic reviews. BM] 1994, 309:|286-129| [http:// www.bmi.com/cgi/content/full/309/6964//286]

47. Crumley ET, Wiebe N, Cramer K, Klassen TP, Hartling L: Which resources should be used to identify RCT/CCTs for systematic reviews: a systematic review. BMC Med Res Methodol 2005, 5:24. [http://www.biomedcentral.com//47|-2288/5/24].

48. Hopewell S, Clarke M, Lefebvre C, Scherer R: Handsearching versus electronic searching to identify reports of randomized trials. Cochrane Database of Methodology Reviews 2002, Issue 42002 [http:// www.mrw.interscience.wiley.com/cochrane/clsysrev/articles/ MR00000I/pdf fs.html]. West Sussex, United Kingdom, John Wiley \& Sons, Ltd

49. McManus RJ, Wilson S, Delaney BC, Fitzmaurice DA, Hyde CJ, Tobias RS, Jowett S, Hobbs FD: Review of the usefulness of contacting other experts when conducting a literature search for systematic reviews. $B M]$ 1998, 3 I7:1562-1563.

50. Scherer RW, Langenberg P, von Elm E: Full publication of results initially presented in abstracts. Cochrane Database of Methodology Reviews 2005, Issue 2. Art. No.: MR000005. 2005 [http://www.mrw.inter science.wiley.com/cochrane/clsysrev/articles/MR000005/pdf fs.html]. West Sussex, United Kingdom, John Wiley \& Sons Ltd

51. Klassen TP, Wiebe N, Russell K, Stevens K, Hartling L, Craig WR, Moher D: Abstracts of randomized controlled trials presented at the society for pediatric research meeting: an example of publication bias. Arch Pediatr Adolesc Med 2002, 156:474-479.

52. Song F, Eastwood AJ, Gilbody S, Duley L, Sutton AJ: Publication and related biases. Health Technol Assess 2000, 4:I-II5 [http:// www.ncchta.org/execsumm/summ4l0.htm]. 
53. Stern JM, Simes RJ: Publication bias: evidence of delayed publication in a cohort study of clinical research projects. $B M J$ 1997, 3 I 5:640-645 [http://bmj.bmijournals.com/cgi/content/full/3/5/ $7109 / 640]$.

54. loannidis JP: Effect of the statistical significance of results on the time to completion and publication of randomized efficacy trials. JAMA 1998, 279:28I-286.

55. Easterbrook PJ, Berlin JA, Gopalan R, Matthews DR: Publication bias in clinical research. Lancet 199|, 337:867-872.

56. Cook DJ, Guyatt GH, Ryan G, Clifton J, Buckingham L, Willan A, Mcllroy $W$, Oxman $A D$ : Should unpublished data be included in meta-analyses? Current convictions and controversies. JAMA 1993, 269:2749-2753.

57. Dundar Y, Dodd S, Dickson R, Walley T, Haycox A, Williamson PR: Comparison of conference abstracts and presentations with full-text articles in the health technology assessments of rapidly evolving technologies. Health Technol Assess 2006, I0:iii- I 45.

58. Hartling L, McAlister FA, Rowe BH, Ezekowitz J, Friesen C, Klassen TP: Challenges in systematic reviews of therapeutic devices and procedures. Ann Intern Med 2005, I 42: I I00-IIIII.

59. Moher D, Tetzlaff J, Tricco AC, Sampson M, Altman DG: Epidemiology and Reporting Characteristics of Systematic Reviews. PLoS Med 2007, 4:e78.

60. McCulloch P, Taylor I, Sasako M, Lovett B, Griffin D: Randomised trials in surgery: problems and possible solutions. BMJ 2002, 324:|448-I45I] [http://bmj.bmijournals.com/cgi/reprint/324/735I/ 1448].

61. Moher D, Schachter HM: Potential solutions to the problem of conducting systematic reviews of new health technologies. CMAJ 2002, I66:1674-1675.

62. Buchkowsky SS, Jewesson PJ: Industry sponsorship and authorship of clinical trials over 20 years. Ann Pharmacother 2004, 38:579-585.

63. Melander H, Ahlqvist-Rastad J, Meijer G, Beermann B: Evidence b(i)ased medicine--selective reporting from studies sponsored by pharmaceutical industry: review of studies in new drug applications. BMJ 2003, 326: I I7I-II73.

64. Lexchin J, Bero LA, Djulbegovic B, Clark O: Pharmaceutical industry sponsorship and research outcome and quality: systematic review. $B M / 2003,326: 1167-1$ I 70.

65. Bekelman JE, Li Y, Gross CP: Scope and impact of financial conflicts of interest in biomedical research: a systematic review. JAMA 2003, 289:454-465.

66. Jorgensen AW, Hilden J, Gotzsche PC: Cochrane reviews compared with industry supported meta-analyses and other meta-analyses of the same drugs: systematic review. $B M J$ 2006, 333:782.

67. Costa V, Brophy J, McGregor M: Vacuum-assisted wound closure therapy $\begin{array}{llll}\text { (V.A.C (R)) 2005:I-24 [http://upload.mcgill.ca/taul } & \end{array}$ VAC REPORT FINAL.pdf]. Montreal, Quebec, Canada, McGill University Health Centre (MUHC) Technology Assessment Unit (TAU)

68. Pham CT, Middleton PF, Maddern GJ: The safety and efficacy of topical negative pressure in non-healing wounds: a systematic review. In J Wound Care Volume 15. England; 2006:240-250.

69. McCallon SK, Knight CA, Valiulus JP, Cunningham MW, McCulloch JM, Farinas LP: Vacuum-assisted closure versus saline-moistened gauze in the healing of postoperative diabetic foot wounds. Ostomy Wound Manage 2000, 46:28-34.

70. Genecov DG, Schneider AM, Morykwas MJ, Parker D, White WL, Argenta LC: A controlled subatmospheric pressure dressing increases the rate of skin graft donor site reepithelialization. Ann Plast Surg 1998, 40:219-225.

71. Negative pressure wound therapy for wound healing Lansdale, Pennsylvania, USA, Hayes, Inc; 2003.

72. Ontario Health Technology Advisory Committee (OHTAC): Vacuum assisted closure therapy for wound care Toronto, Ontario, Canada, Ministry of Health and Long-Term Care (MOHLTC) Medical Advisory Secretariat (MAS); 2004:I-59.

73. Pham C, Middleton P, Maddern G: Vacuum-assisted closure for the management of wounds: an accelerated systematic review. 2003 [http:// www.surgeons.org/asernip-s/publications vacuum.htm]. Adelaide, Australia, Australian safety and efficacy register of new interventional procedures - surgical (ASERNIP-S)

74. Vuerstaek JDD, Wuite J, Neuman H, Steijlen P, Veraart JCJM: The management of recalcitrant leg ulcers. Abstract P029. In Presented at the 2nd World Union of Wound Healing Societies Meeting 2004
(July 8-I3), Paris, France Paris, France, Second World Union of Wound Healing Societies Meeting (08-13 July); 2004.

75. Obdeijn MC, Braakenburg A, Feit R, Klinkenbul: Vac therapy versus hydrocolloids in acute and chronic wounds. Abstract EO I 2. In Presented at the 2nd World Union of Wound Healing Societies Meeting 2004 (July 8-13), Paris, France Paris, France, Second World Union of Wound Healing Societies Meeting (08-13 July); 2004.

76. Payne W: VAC therapy vs. moist wound therapy in the treatment of diabetic foot amputation wounds: preliminary results of a multicenter trial. Abstract EOI I. In Presented at the 2nd World Union of Wound Healing Societies Meeting 2004 (July 8-13), Paris, France Paris, France, Second World Union of Wound Healing Societies Meeting (08-13 July); 2004.

77. Moues CM, Van Den Bemd GJCM, Heule F, Hovius SER: A prospective randomized trial comparing vacuun therapy to conventional moist gauze therapy. Abstract A00 I. In Presented at the 2nd World Union of Wound Healing Societies Meeting 2004 (July 8-13), Paris, France Paris, France, Second World Union of Wound Healing Societies Meeting (08-13 July); 2004.

78. Heath T, Moisidis E, Deva A: A prospective controlled trial of vacuum assisted closure (VAC) in the treatment of acute surgical wounds requiring split skin grafting. Abstract. In Adelaide, Australia, Fourth Australian Wound Management Association Conference (March); 2002.

79. Joseph E, Hamori CA, Bergman S, Roaf C, Swan N: A prospective randomized trial of vacuum-assisted closure versus standard therapy of chronic nonhealing wounds. Abstract. Denver, Colorado, USA, I4th Annual Clinical Symposium on Wound Care (30 September - 04 October); 1999.

80. Stannard JP, Robinson JT, Anderson ER, McGwin GJ, Volgas DA, Alonso JE: Negative pressure wound therapy to treat hematomas and surgical incisions following high-energy trauma. In J Trauma Volume 60. United States; 2006: I 30 I-I 306.

8I. Buttenschön K, Fleischmann W, Haupt U, Kinzl L, Buttenschön DC: The influence of vacuum-assisted closure on inflammatory tissue reactions in the postoperative course of ankle fractions. Foot Ankle Surg 200I, 7:165-173.

82. Jeschke MG, Rose C, Angele P, Fuchtmeier B, Nerlich MN, Bolder U: Development of new reconstructive techniques: use of Integra in combination with fibrin glue and negative-pressure therapy for reconstruction of acute and chronic wounds. In Plast Reconstr Surg Volume I I 3. United States; 2004:525-530.

83. Evans D, Land L: Topical negative pressure for treating chronic wounds. The Cochrane Database of Systematic Reviews. Art. No.: CD00 1898. DOI: 10.1002//465/858. 200I [http://www.mrw.interscience.wiley.com/ cochrane/clsysrev/articles/CD001898/pdf fs.html]. West Sussex, United Kingdom, John Wiley \& Sons Ltd

84. Fisher A, Brady B: Vacuum assisted wound closure therapy. 2003 [http:/ /cadth.ca/media/pdf/22I vac cetap e.pdf]. Ottawa, Ontario, Canada, The Canadian Coordinating Office for Health Technology Assessment (CCOHTA)

85. Higgins S: The effectiveness of vacuum assisted closure (VAC) in wound healing 2003 [http://www.mihsr.monash.org/cce/res/pdf/c/99/fr.pdf]. Clayton, Australia, Centre for Clinical Effectiveness (CCE)

\section{Pre-publication history}

The pre-publication history for this paper can be accessed here:

http://www.biomedcentral.com/1471-2288/8/4/prepub 